\title{
Existence of Ground States and KMS States for Approximately Inner Dynamics ${ }^{\star}$
}

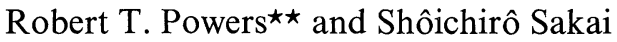 \\ University of Pennsylvania, Philadelphia, Pennsylvania, USA \\ Received July 1, 1974; in revised form August 5, 1974
}

\begin{abstract}
A strongly continuous one parameter group of $*$-automorphisms of a $C^{*}$-algebra with unit is said to be approximately inner if it can be approximated strongly by inner one parameter groups of *-automorphisms. It is shown that an approximately inner one parameter group of $*$-automorphisms has a ground state and, if there exists a trace state, a KMS state for all inverse temperatures. It follows that quantum lattice systems have ground states and KMS states. Conditions that a strongly continuous one parameter group of *-automorphisms of a UHF algebra be approximately inner are given in terms of the unbounded derivation which generates the automorphism group.
\end{abstract}

\section{Introduction}

Suppose $\left\{\alpha_{t} ;-\infty<t<\infty\right\}$ is a strongly continuous one parameter group of *-automorphisms of a $C^{*}$-algebra $\mathfrak{A}$ with unit, where by strongly continuous we mean $\left\|\alpha_{t}(A)-A\right\| \rightarrow 0$ as $t \rightarrow 0$ for each $A \in \mathfrak{A}$. We say the group $\left\{\alpha_{t}\right\}$ is approximately inner if there exists a sequence $\left\{H_{n}\right\}$ of hermitian elements of $\mathfrak{A}$ such that

$$
\left\|e^{i t H_{n}} A e^{-i t H_{n}}-\alpha_{t}(A)\right\| \rightarrow 0
$$

as $n \rightarrow \infty$ for each $A \in \mathfrak{A}$ where for fixed $A$ the convergence is uniform for $t$ in a compact set. In this paper we will show that if $\left\{\alpha_{t}\right\}$ is approximately inner then there exists at least one ground state (Section 2) and there exist $K M S$ states for all inverse temperatures $\beta$ (Section 3) provided $\mathfrak{U}$ has a trace state. Since for quantum lattice systems the dynamics is given by approximately inner one parameter groups of *-automorphisms (see e.g. ([14], p. 193), [13] or [1]) it follows that quantum lattice systems have ground states and KMS states for all inverse temperatures $\beta$. Ruelle has shown the existence of ground states for quantum lattice systems in [15, Theorems 2(c) and 4].

In working with a strongly continuous one parameter group of *-automorphisms $\left\{\alpha_{t}\right\}$ it is often useful to introduce the unbounded derivation $\delta$ which generates the group. Suppose $\left\{\alpha_{t}\right\}$ is a strongly

* This research is supported in part by a National Science Foundation Grant.

$\star \star$ Alfred P. Sloan Fellow. 
continuous group of $*$-automorphisms of a $C^{*}$-algebra $\mathfrak{A}$. The generator of the group $\left\{\alpha_{t}\right\}$ is a derivation $\delta$ given by

$$
\delta(A)=\lim _{t \rightarrow 0}\left(\alpha_{t}(A)-A\right) / t
$$

where the domain $\mathfrak{D}(\delta)$ of $\delta$ is the linear manifold of all $A \in \mathfrak{U}$ such that the above limit exists in the sense of norm convergence. It follows from semigroup theory (see [3] or [8]) and the fact the $\alpha_{t}$ are $*$-automorphisms that $\delta$ has the properties,

(i) $\mathfrak{D}(\delta)$ is a norm dense linear subset of $\mathfrak{A}$ and $\delta$ is a linear mapping of $\mathfrak{D}(\delta)$ into $\mathfrak{U}$.

(ii) $\mathfrak{D}(\delta)$ is an algebra and if $A, B \in \mathfrak{D}(\delta)$ then $A B \in \mathfrak{D}(\delta)$ and $\delta(A B)=\delta(A) B+A \delta(B)$.

(iii) $\mathfrak{D}(\delta)$ is a *-algebra and if $A \in \mathfrak{D}(\delta)$ then $A^{*} \in \mathfrak{D}(\delta)$ and $\delta\left(A^{*}\right)=\delta(A)^{*}$.

(iv) $\delta$ is closed, i.e., if $A_{n} \in \mathfrak{D}(\delta),\left\|A_{n}-A\right\| \rightarrow 0$ and $\left\|\delta\left(A_{n}\right)-B\right\| \rightarrow 0$ as $n \rightarrow \infty$ then $A \in \mathfrak{D}(\delta)$ and $\delta(A)=B$.

Recently it was shown in [17] that if $\left\{\alpha_{t}\right\}$ is a strongly continuous one parameter group of $*$-automorphism of a UHF-algebra $\mathfrak{A}$ then there is an increasing sequence $M_{1} \subset M_{2} \subset \cdots$ of $\left(n_{i} \times n_{i}\right)$-matrix algebras so that $\mathfrak{U}_{0}=\bigcup_{i=1}^{\infty} M_{i}$ is a norm dense $*$-subalgebra of $\mathfrak{U}$ and each element $A \in \mathfrak{U}_{0}$ is an analytic element for $\delta$ the generator of $\left\{\alpha_{t}\right\}$ [i.e., if $A \in \mathfrak{U}_{0}$ then $\alpha_{t}(A)$ can be extended to an analytic function which is holomorphic for $|\operatorname{Im}(t)|<r_{0}$ with $\left.r_{0}>0\right]$. Furthermore, it was shown that there exists a sequence of hermitian elements $H_{n} \in \mathfrak{U}$ so that $i\left[H_{n}, A\right]=\delta(A)$ for all $A \in M_{n}$. It follows that if $A \in \mathfrak{U}_{0}$ we have $\delta(A)=\lim _{n \rightarrow \infty} i\left[H_{n}, A\right]$. We will show in Section 4 that if $\mathfrak{U}_{0}$ is a core for $\delta$ then $\left\{\alpha_{t}\right\}$ is approximately inner.

We end the paper with the conjecture that all strongly continuous one parameter groups of *-automorphisms of UHF-algebras are approximately inner. It would follow from the truth of this conjecture that all strongly continuous one parameter groups of $*$-automorphisms of UHF-algebras have ground states and KMS states for all inverse temperatures $\beta$.

\section{Existence of Ground States}

We begin this section with the definition of a ground state on a $C^{*}$-algebra with respect to a one parameter group of $*$-automorphisms. This definition is essentially the spectral condition of quantum field theory (see ([20], Chapter 3) and [2]).

Definition 2.1. Suppose $\left\{\alpha_{t}\right\}$ is a one parameter group of $*$-automorphisms of a $C^{*}$-algebra $\mathfrak{U}$ with unit. We say $\omega$ is a ground state of 
$\mathfrak{U}$ for the group $\left\{\alpha_{t}\right\}$ if $\omega$ is a state of $\mathfrak{U}$ with the property, if $A, B \in \mathfrak{U}$ then $\omega\left(A \alpha_{t}(B)\right)$ is a continuous function of $t$ and

$$
\int h(t) \omega\left(A \alpha_{t}(B)\right) d t=0
$$

for all continuous $L^{1}$-functions $h$ whose Fourier transform

$$
\tilde{h}(\lambda)=\frac{1}{\sqrt{2 \pi}} \int e^{-i t \lambda} h(t) d t
$$

vanishes on the negative real axis $(-\infty, 0]$.

We remark that a ground state $\omega$ for $\left\{\alpha_{t}\right\}$ is necessarily $\alpha_{t}$ invariant. To see this note that if $A=A^{*} \in \mathfrak{A}$ then the function $h(t)=\omega\left(\alpha_{t}(A)\right)$ defines a tempered distribution (see [4] or [18]) by the relation

$$
T(f)=\int f(t) h(t) d t
$$

for all $f$ in Schwartz's space. Since $\omega$ is a ground state the Fourier transform $\tilde{T}$ of $T$ has support on the positive real axis $[0, \infty)$. Since $h$ is a real valued function $T$ is real, i.e., $\overline{T(t)}=T(t)$ for all $t$, and, therefore we have $\bar{T}(k)$ $=\tilde{T}(-k)$ for the Fourier transform. Hence, $\tilde{T}$ has support on the negative real axis $(-\infty, 0]$. Thus, $\tilde{T}$ has its support at the single point 0 . From the theory of distributions (see [4] or [18]) it follows that $\tilde{T}$ is a finite sum of derivatives of $\delta$-functions at zero, i.e., $\tilde{T}(k)=\sum_{n=0}^{m} a_{n} \delta^{(n)}(k)$ and hence $T(t)=(2 \pi)^{-1 / 2} \sum_{n=0}^{m} a_{n}(-i t)^{n}$. Since $h$ is a bounded function we have $T(t)=h(t)=a_{0} / \sqrt{2 \pi}$ a constant. Hence $\omega\left(\alpha_{t}(A)\right)=\omega(A)$ for all $t$ and all hermitian $A \in \mathfrak{A}$. Hence, $\omega$ is $\alpha_{t}$ invariant.

The fact that $\omega$ is a ground state has the following implications for the *-representation induced by $\omega$. Suppose $\omega$ is an $\alpha_{t}$ invariant state of $\mathfrak{U}$ and $\left(\pi, \mathfrak{H}, f_{0}\right)$ is a cyclic $*$-representation of $\mathfrak{U}$ induced by $\omega$ on a Hilbert space $\mathfrak{H}$ with cyclic vector $f_{0}$ so that $\omega(A)=\left(f_{0}, \pi(A) f_{0}\right)$ for all $A \in \mathfrak{U}$. Since $\omega$ is $\alpha_{t}$ invariant we may define unitary operators $U(t)$ on $\mathfrak{H}$ by the relations

$$
U(t) \pi(A) f_{0}=\pi\left(\alpha_{t}(A)\right) f_{0}
$$

for all $A \in \mathfrak{A}$. One can easily check that the above relations uniquely define isometries $U(t)$ of $\mathfrak{H}$ into $\mathfrak{H}$. From the group property of $\alpha_{t}$ and the continuity of $\omega\left(A^{*} \alpha_{t}(A)\right)$ in $t$ for all $A \in \mathfrak{A}$ it follows that $t \rightarrow U(t)$ is a strongly continuous one parameter group of unitary operators with the additional properties,

and

$$
U(t) \pi(A) U(t)^{-1}=\pi\left(\alpha_{t}(A)\right)
$$

for all real $t$ and all $A \in \mathfrak{A}$.

$$
U(t) f_{0}=f_{0}
$$


From Stone's theorem (see e.g. $([12]$, Chapter X)) it follows there is a self-adjoint operator $H$ which generates the one parameter group $U(t)=e^{i t H}$. Since $U(t) f_{0}=f_{0}$ for all real $t$ we have $f_{0}=\mathfrak{D}(H)$, the domain of $H$, and $H f_{0}=0$. Let $\{E(\lambda) ;-\infty<\lambda<\infty\}$ be the spectral resolution of $H$, i.e.,

$$
H=\int d E(\lambda) \text { and } U(t)=\int e^{i t \lambda} d E(\lambda) .
$$

For $A, B \in \mathfrak{A}$ and $h$ an $L^{1}$-function we have

$$
\begin{aligned}
\int h(t) \omega\left(A \alpha_{t}(B)\right) d t & =\int h(t)\left(f_{0}, \pi(A) U(t) \pi(B) f_{0}\right) d t \\
& =\int h(t) \int e^{i t \lambda}\left(\pi\left(A^{*}\right) f_{0}, d E(\lambda) \pi(B) f_{0}\right) d t \\
& =\sqrt{2 \pi} \int \tilde{h}(-\lambda)\left(\pi\left(A^{*}\right) f_{0}, d E(\lambda) \pi(B) f_{0}\right)
\end{aligned}
$$

where in the last equality we have carried out the $t$ integration. We have $\omega$ is a ground state if and only if the above integral vanishes for all $A, B \in \mathfrak{A}$ provided $\tilde{h}$ vanishes on the negative real axis $(-\infty, 0]$. Since $\left\{\pi\left(A^{*}\right) f_{0}\right.$; $A \in \mathfrak{U}\}$ and $\left\{\pi(B) f_{0} ; B \in \mathfrak{U}\right\}$ are dense in $\mathfrak{H}$ we have the fact that $\omega$ is a ground state is equivalent to the fact that the spectral measure $E(\lambda)$ has its support on the positive real axis $[0, \infty)$. Hence, $\omega$ is a ground state if and only if $H$ is positive, i.e., $H \geqq 0$.

If $\omega$ is a ground state and if we associate the self-adjoint operator $H$ with the energy of a physical system then the vector $f_{0}$ is a vector of norm one which minimizes the energy $(f, H f)$ with $\|f\|=1$. This is the origin of the term "ground state" for the state $\omega(A)=\left(f_{0}, \pi(A) f_{0}\right)$ for all $A \in \mathfrak{A}$.

The following theorem may be useful in characterizing ground states in terms of unbounded derivations.

Theorem 2.2. Suppose $\left\{\alpha_{t}\right\}$ is a strongly continuous one parameter group of *-automorphisms of a $C^{*}$-algebra $\mathfrak{U}$ with unit. Suppose $\delta$ is the generator of $\left\{\alpha_{t}\right\}$ and $\mathfrak{D}$ is a core for $\delta$. Then, a state $\omega$ is a ground state for $\left\{\alpha_{t}\right\}$ if and only if

$$
-i \omega\left(A^{*} \delta(A)\right) \geqq 0
$$

for all $A \in \mathfrak{D}$.

Proof. First suppose $\omega$ is a ground state for $\left\{\alpha_{t}\right\}$. Let $\left(\pi, \mathfrak{H}, f_{0}\right)$ be a cyclic *-representation of $\mathfrak{U}$ induced by $\omega$ with cyclic vector $f_{0} \in \mathfrak{H}$ so that $\omega(A)=\left(f_{0}, \pi(A) f_{0}\right)$ for all $A \in \mathfrak{U}$. We have from the previous discussion that there is a strongly continuous one parameter group of unitary operators $U(t)=e^{i t H}$ with $U(t) f_{0}=f_{0}$ and

$$
U(t) \pi(A) U(t)^{-1}=\pi\left(\alpha_{t}(A)\right)
$$

for all real $t$ and $A \in \mathfrak{A}$. Since $\omega$ is a ground state we have that the generator $H$ of $\{U(t)\}$ is positive, i.e., $H \geqq 0$. 
Now if $A \in \mathfrak{D}(\delta)$ we have

$$
(i t)^{-1}(U(t)-I) \pi(A) f_{0}=(i t)^{-1} \pi\left(\alpha_{t}(A)-A\right) f_{0} \rightarrow-i \pi(\delta(A)) f_{0}
$$

as $t \rightarrow 0$. Hence, from Stone's theorem we have $\pi(A) f_{0} \in \mathfrak{D}(H)$, the domain of $H$, and $H \pi(A) f_{0}=-i \pi(\delta(A)) f_{0}$. Now, if $A \in \mathfrak{D} \subset \mathfrak{D}(\delta)$ we have

$$
\begin{aligned}
-i \omega\left(A^{*} \delta(A)\right) & =-i\left(f_{0}, \pi\left(A^{*}\right) \pi(\delta(A)) f_{0}\right)=\left(f_{0}, \pi\left(A^{*}\right) H \pi(A) f_{0}\right) \\
& =\left(\pi(A) f_{0}, H \pi(A) f_{0}\right) \geqq 0 .
\end{aligned}
$$

Hence, if $\omega$ is a ground state for $\left\{\alpha_{t}\right\}$ then $-i \omega\left(A^{*} \delta(A)\right) \geqq 0$ for all $A \in \mathfrak{D} \subset \mathfrak{D}(\delta)$.

Next suppose $\omega$ is a state of $\mathfrak{A}$ so that $-i \omega\left(A^{*} \delta(A)\right) \geqq 0$ for all $A \in \mathfrak{D} \subset \mathfrak{D}(\delta)$ where $\mathfrak{D}$ is a core for $\delta$. We first show $-i \omega\left(A^{*} \delta(A)\right) \geqq 0$ for all $A \in \mathfrak{D}(\delta)$. Since $\mathfrak{D}$ is a core for $\delta$ there is for each $A \in \mathfrak{D}(\delta)$ a sequence $\left\{A_{n} \in \mathfrak{D}\right\}$ so that $\left\|A_{n}-A\right\| \rightarrow 0$ and $\left\|\delta\left(A_{n}\right)-\delta(A)\right\| \rightarrow 0$ as $n \rightarrow \infty$. Since multiplication is jointly continuous we have $\left\|A_{n}^{*} \delta\left(A_{n}\right)-A^{*} \delta(A)\right\| \rightarrow 0$ as $n \rightarrow \infty$. Hence, if $A \in \mathfrak{D}(\delta)$ we have

$$
-i \omega\left(A^{*} \delta(A)\right)=\lim _{n \rightarrow \infty}-i \omega\left(A_{n}^{*} \delta\left(A_{n}\right)\right) \geqq 0 .
$$

Hence, $-i \omega\left(A^{*} \delta(A)\right) \geqq 0$ for all $A \in \mathfrak{D}(\delta)$.

Next we will show $\omega$ is $\alpha_{t}$ invariant. Since $\alpha_{t}(I)=I$ for all real $t$ it follows that $I \in \mathfrak{D}(\delta)$ and $\delta(I)=0$. If $A \in \mathfrak{D}(\delta)$ and $\lambda$ is a complex number we have $-i \omega\left((\lambda I+A)^{*} \delta(\lambda I+A)\right) \geqq 0$. Hence, we have $-i \omega(\bar{\lambda} \delta(A)$ $\left.+A^{*} \delta(A)\right) \geqq 0$ for all complex $\lambda$. Hence, $\omega(\delta(A))=0$ for all $A \in \mathfrak{D}(\delta)$. Since $\alpha_{t}$ maps $\mathfrak{D}(\delta)$ into $\mathfrak{D}(\delta)$ we have for all $A \in \mathfrak{D}(\delta)$

$$
\frac{d}{d t} \omega\left(\alpha_{t}(A)\right)=\omega\left(\delta\left(\alpha_{t}(A)\right)\right)=0 \text {. }
$$

Hence, $\omega\left(\alpha_{t}(A)\right)=\omega(A)$ for real $t$ and $A \in \mathfrak{D}(\delta)$. Since $\mathfrak{D}(\delta)$ is norm dense in $\mathfrak{A}$ we have $\omega$ is $\alpha_{t}$ invariant.

Let $\left(\pi, \mathfrak{H}, f_{0}\right)$ be the cyclic $*$-representation induced by $\omega$ and let $t \rightarrow U(t)$ be the strongly continuous one parameter unitary group defined by the relations

$$
U(t) \pi(A) f_{0}=\pi\left(\alpha_{t}(A)\right) f_{0}
$$

for $t$ real and all $A \in \mathfrak{A}$. Let $H$ be the generator of the group $\{U(t)\}$, i.e., $U(t)=e^{i t H}$. To prove $\omega$ is a ground state we must show $H \geqq 0$. Suppose $A \in \mathfrak{D}(\delta)$. Then, we have

$$
(i t)^{-1}(U(t)-I) \pi(A) f_{0}=(i t)^{-1} \pi\left(\alpha_{t}(A)-A\right) f_{0} \Rightarrow-i \pi(\delta(A)) f_{0}
$$

as $t \rightarrow 0$. 
Hence, from Stone's theorem we have $\pi(A) f_{0} \in \mathfrak{D}(H)$ and $H \pi(A) f_{0}$ $=-i \pi(\delta(A)) f_{0}$ for all $A \in \mathfrak{D}(\delta)$. We have for $A \in \mathfrak{D}(\delta)$

$$
\begin{aligned}
\left(\pi(A) f_{0}, H \pi(A) f_{0}\right) & =-i\left(\pi(A) f_{0}, \pi(\delta(A)) f_{0}\right) \\
& =-i\left(f_{0}, \pi\left(A^{*} \delta(A)\right) f_{0}\right) \\
& =-i \omega\left(A^{*} \delta(A)\right) \geqq 0 .
\end{aligned}
$$

Let $H_{1}$ be the closure of the restriction of $H$ to $\left\{\pi(\mathfrak{D}(\delta)) f_{0}\right\}$, i.e., $H_{1}=\overline{H \mid\left\{\pi(\mathfrak{D}(\delta)) f_{0}\right\}}$. From the above inequality we have $H_{1}$ is positive. We will show $H$ is positive by showing $H_{1}=H$.

Since $H_{1}$ is a restriction of $H$ (i.e., $H_{1} \subset H$ ) we have $H_{1}^{*}$ is an extension of $H^{*}=H$. Hence, we have $H_{1} \subset H \subset H_{1}^{*}$. We will show $H_{1}=H_{1}^{*}$ thereby showing $H=H_{1}$.

We have $\left.U(t)\left\{\pi(\mathfrak{D}(\delta)) f_{0}\right\}=\left\{\pi\left(\alpha_{t}(\mathfrak{D}(\delta))\right)\right\} f_{0}\right\}=\left\{\pi(\mathfrak{D}(\delta)) f_{0}\right\}$ since $\mathfrak{D}(\delta)$ is invariant under $\alpha_{t}$. Since $\left\{\pi(\mathfrak{D}(\delta)) f_{0}\right\}$ is a dense linear manifold of $\mathfrak{D}\left(H_{1}\right)$ invariant under $U(t)$ it follows from Lemma 2 of [19] that $H_{1}$ is self-adjoint. Hence, $H=H_{1}$ is positive and $\omega$ is a ground state. This completes the proof of the theorem.

Theorem 2.3. Suppose $\left\{\alpha_{t}\right\}$ is a strongly continuous one parameter group of *-automorphisms of a $C^{*}$-algebra $\mathfrak{A}$ with unit. Suppose $\left\{\alpha_{t}\right\}$ is approximately inner. Then, there exists a ground state $\omega$ for $\left\{\alpha_{t}\right\}$. The ground state need not be unique.

Proof. Suppose the hypothesis of the theorem are satisfied. Since $\left\{\alpha_{t}\right\}$ is approximately inner there is a sequence of hermitian elements $\left\{H_{n} \in \mathfrak{U}\right\}$ so that $\left\|e^{i t H_{n}} A e^{-i t H_{n}}-\alpha_{t}(A)\right\| \rightarrow 0$ as $n \rightarrow \infty$ for all $A \in \mathfrak{U}$ where for fixed $A \in \mathfrak{U}$ the convergence is uniform on compact sets. By adding a multiple of the unit to $H_{n}$ we can arrange it so that $H_{n}$ is positive and zero is in the spectrum of $H_{n}$, i.e., $0 \in \sigma\left(H_{n}\right)$ and $H_{n} \geqq 0$ for $n=1,2, \ldots$ Since $0 \in \sigma\left(H_{n}\right)$ it follows from $\left([16]\right.$ or $\left([10]\right.$, p. 306) there is a state $\omega_{n}$ of $\mathfrak{U}$ so that $\omega_{n}\left(H_{n}\right)=\omega_{n}\left(H_{n}^{2}\right)=0$. Since the state space of a $C^{*}$-algebra is compact in the weak *-topology there is a state $\omega$ which is a cluster point of the sequence $\left\{\omega_{n}\right\}$ in the weak $*$-topology. We will show $\omega$ is a ground state.

Suppose $h$ is a continuous $L^{1}$-function whose Fourier transform $\tilde{h}$ vanishes on the negative real axis $(-\infty, 0]$. Suppose $A, B \in \mathfrak{A}$. We will show

Let

$$
\int h(t) \omega\left(A \alpha_{t}(B)\right) d t=0 .
$$

$$
B_{0}=\int h(t) \alpha_{t}(B) d t \quad \text { and } \quad B_{n}=\int h(t) e^{i t H_{n}} A e^{-i t H_{n}} d t .
$$

Suppose $\varepsilon>0$. Since $h \in L^{1}$ there is a constant $c$ so that

$$
2\|B\| \int_{|t|>c}|h(t)| d t<\varepsilon / 2 .
$$


Since $e^{i t H_{n}} B e^{-i t H_{n}}$ converges to $\alpha_{t}(B)$ uniformly for $|t| \leqq c$ there is an integer $n_{0}$ so that $\|h\|_{1}\left\|e^{i t H_{n}} B e^{-i t H_{n}}-\alpha_{t}(B)\right\|<\varepsilon / 2$ for $|t| \leqq c$ and $n \geqq n_{0}$ where $\|h\|_{1}$ is the $L^{1}$ norm of $h$. For $n \geqq n_{0}$ we have

$$
\begin{aligned}
\left\|B_{n}-B_{0}\right\| \leqq & \left\|\int h(t)\left(e^{i t \boldsymbol{H}_{n}} B e^{-i t \boldsymbol{H}_{n}}-\alpha_{t}(B)\right) d t\right\| \\
\leqq & \int_{-c}^{+c}|h(t)|\left\|e^{i t \boldsymbol{H}_{n}} B e^{-i t \boldsymbol{H}_{n}}-\alpha_{t}(B)\right\| d t \\
& +\int_{|t|>c}|h(t)|\left\|e^{i t H_{n}} B e^{-i t H_{n}}-\alpha_{t}(B)\right\| d t \\
\leqq & \int_{-c}^{c}|h(t)|\left(\|h\|_{1}\right)^{-1}(\varepsilon / 2) d t+2\|B\| \int_{|t|>c}|h(t)| d t \\
\leqq & \varepsilon / 2+\varepsilon / 2=\varepsilon .
\end{aligned}
$$

Since $\omega$ is a cluster point of the sequence $\left\{\omega_{n}\right\}$ in the weak $*$-topology there is an integer $r \geqq n_{0}$ so that $\left|\omega_{r}\left(A B_{0}\right)-\omega\left(A B_{0}\right)\right|<\varepsilon$. Now, we have

$$
\begin{aligned}
\omega_{r}\left(A B_{r}\right) & =\int h(t) \omega_{r}\left(A e^{i t H_{r}} B e^{-i t H_{r}}\right) d t \\
& =\int h(t) \omega_{r}\left(A e^{i t H_{r}} B\right) d t \\
& =\sqrt{2 \pi} \omega_{r}\left(A \tilde{h}\left(-H_{r}\right) B\right)=0
\end{aligned}
$$

where we have $\tilde{h}\left(-H_{r}\right)=0$ since the Fourier transform $\tilde{h}$ of $h$ is a continuous function which vanishes on the negative real axis $(-\infty, 0]$ and the spectrum of $-H_{r}$ is contained in this interval. Hence, we have

$$
\begin{aligned}
\left|\omega\left(A B_{0}\right)\right| & \leqq\left|\omega\left(A B_{0}\right)-\omega_{r}\left(A B_{0}\right)\right|+\left|\omega_{r}\left(A B_{0}\right)-\omega_{r}\left(A B_{r}\right)\right|+\left|\omega_{r}\left(A B_{r}\right)\right| \\
& \leqq \varepsilon+\left\|A\left(B_{0}-B_{r}\right)\right\|+0 \\
& \leqq \varepsilon+\|A\| \varepsilon .
\end{aligned}
$$

Since $\varepsilon>0$ is arbitrary we have

$$
\omega\left(A B_{0}\right)=\int h(t) \omega\left(A \alpha_{t}(B)\right) d t=0 .
$$

Hence, $\omega$ is a ground state. This completes the proof of the theorem.

Remark. Since for quantum lattice systems the dynamics is given by an approximately inner one parameter group of *-automorphisms (see e.g. ([14], p. 193), [13] and [1]), it follows that quantum lattice systems have ground states. Ruelle has shown the existence of ground states for quantum lattice systems in [15, Theorems 2(c) and 4]. We thank the referee for pointing out this reference to us. 


\section{Existence of KMS States}

We begin this section with the definition of $\operatorname{KMS}$ states (see $[14,13]$ and [6]).

Definition 3.1. Suppose $\left\{\alpha_{t}\right\}$ is a one parameter group of $*$-automorphisms of a $C^{*}$-algebra $\mathfrak{U}$ with unit. We say $\omega$ is a KMS state for $\left\{\alpha_{t}\right\}$ of inverse temperature $\beta>0$ if for each $A, B \in \mathfrak{U}$ there exists an analytic function $F$ which is holomorphic for $0<\operatorname{Im}(z)<\beta$ and continuous for $0 \leqq \operatorname{Im}(z) \leqq \beta$ so that

$$
\omega\left(A \alpha_{t}(B)\right)=F(t) \quad \text { and } \quad \omega\left(\alpha_{t}(A) B\right)=F(t+i \beta)
$$

for all real $t$.

As in the case of ground states it follows that if $\omega$ is a KMS state for $\left\{\alpha_{t}\right\}$ then $\omega$ is $\alpha_{t}$ invariant. It is thought that KMS states describe physical systems in thermal equilibrium where the dynamics is given by the *-automorphism group $\left\{\alpha_{t}\right\}$ (see [14]).

Theorem 3.2. Suppose $\left\{\alpha_{t}\right\}$ is a strongly continuous one parameter group of *-automorphisms of a $C^{*}$-algebra $\mathfrak{A}$ with unit. Suppose $\left\{\alpha_{t}\right\}$ is approximately inner. Furthermore, suppose $\mathfrak{U}$ has one or more trace states $\tau$ [i.e., $\tau(A B)=\tau(B A)$ for all $A, B \in \mathfrak{A}]$. Then, there exists at least one $\mathrm{KMS}$ state $\omega_{\beta}$ for all inverse temperatures $\beta>0$.

Proof. Suppose the hypothesis of the theorem is satisfied and $\pi$ is a trace state of $\mathfrak{U}$ and $\beta>0$. Since $\left\{\alpha_{t}\right\}$ is approximately inner there is a sequence of hermitian elements $\left\{H_{n} \in \mathfrak{A}\right\}$ so that $\left\|e^{i t H_{n}} A e^{-i t H_{n}}-\alpha_{t}(A)\right\| \rightarrow 0$ as $n \rightarrow \infty$ for each fixed $A \in \mathfrak{U}$ where the convergence is uniform for $t$ in a compact set. Let $\omega_{n}(A)=\tau\left(e^{-\beta H_{n}} A\right) / \tau\left(e^{-\beta H_{n}}\right)$ for all $A \in \mathfrak{A}$. A straight forward computation shows that the $\omega_{n}$ are states of $\mathfrak{A}$ which satisfy the KMS condition for the automorphism groups $\alpha_{t}^{(n)}(A)=e^{i t H_{n}} A e^{-i t H_{n}}$ for $A \in \mathfrak{U}$. Since the state space of a $C^{*}$-algebra with unit is compact in the weak $*$-topology there is a state $\omega$ which is a cluster point of the sequence $\left\{\omega_{n}\right\}$ in the weak $*$-topology. We will show $\omega$ satisfies the KMS condition.

Suppose $A, B \in \mathfrak{A}$. Let $\mathfrak{U}_{0}$ be the smallest $C^{*}$-subalgebra of $\mathfrak{A}$ containing $\left\{A, \alpha_{t}(B), H_{n} ;-\infty<t<\infty, n=1,2, \ldots\right\}$. Since $\alpha_{t}(B)$ is norm continuous in $t, \mathfrak{U}_{n}$ is norm separable. Hence, there is a subsequence $\left\{\omega_{n(k)}\right\}$ of the sequence $\left\{\omega_{n}\right\}$ which converges weakly to $\omega$ on $\mathfrak{U}_{0}$ as $k \rightarrow \infty$. Let

$$
F_{k}(z)=\omega_{(n)(k)}\left(A e^{i z H_{n(k)}} B e^{\left.-i z H_{n(k)}\right)} .\right.
$$

We have $F_{k}$ is an entire analytic function which is bounded in the strip $0 \leqq \operatorname{Im}(z) \leqq \beta$. Since analytic functions are harmonic we can express $F_{k}(z)$ for $0 \leqq \operatorname{Im}(z) \leqq \beta$ in terms of $F_{k}(z)$ on the $\operatorname{lines} \operatorname{Im}(z)=0$ and $\operatorname{Im}(z)=\beta$, i.e.,

$$
F_{k}(z)=\int K_{1}(t, z) f_{1 k}(t) d t+\int K_{2}(t, z) f_{2 k}(t) d t
$$


for $0<\operatorname{Im}(z)<\beta$ where

$$
\begin{aligned}
& f_{1 k}(t)=F_{k}(t)=\omega_{n(k)}\left(A e^{i t H_{n(k)}} B e^{-i t H_{n(k)}}\right) \\
& f_{2 k}(t)=F_{k}(t+i \beta)=\omega_{n(k)}\left(e^{i t H_{n(k)}} A e^{-i t H_{n(k)}} \beta\right)
\end{aligned}
$$

for all real $t$ (here in the second equation we have used the fact that $\omega_{n(k)}$ satisfies the KMS condition). The functions $K_{1}$ and $K_{2}$ are positive $L^{1}$-functions of $t$ for each fixed $z$ and $\left\|K_{1}(\cdot, z)\right\|_{1}+\left\|K_{2}(\cdot, z)\right\|_{1}=1$ for all $0<\operatorname{Im}(z)<\beta$ where $\left\|K_{1}(\cdot, z)\right\|_{1}$ is the $L^{1}$ norm of the function $h(t)=K_{1}(t, z), i=1,2$ (see [7], section 18.2).

We wish to thank Professor R. Herman for pointing out to us this integral representation of a function harmonic in the strip and there by greatly simplifying the proof of this theorem.

We have

$$
\begin{aligned}
\left|\omega\left(A \alpha_{t}(B)\right)-f_{1 k}(t)\right| \leqq & \left|\omega\left(A \alpha_{t}(B)\right)-\omega_{n(k)}\left(A \alpha_{t}(B)\right)\right| \\
& +\left|\omega_{n(k)}\left(A \alpha_{t}(B)\right)-\omega_{n(k)}\left(A e^{i t H_{n(k)}} B e^{\left.-i t H_{n(k)}\right)}\right)\right| \\
\leqq & \left|\omega\left(A \alpha_{t}(B)\right)-\omega_{n(k)}\left(A \alpha_{t}(B)\right)\right| \\
& +\|A\|\left\|\alpha_{t}(B)-e^{i t H_{n(k)}} B e^{-i t H_{n(k)}}\right\| .
\end{aligned}
$$

Since $\left\{A \alpha_{t}(B)\right\}$ is norm compact for $t$ in a compact set and $\omega_{n(k)}$ converges weakly to $\omega$ on $\mathfrak{A}_{0}$ we have $\left|\omega\left(A \alpha_{t}(B)\right)-\omega_{n(k)}\left(A \alpha_{t}(B)\right)\right|$ tends to zero uniformly on compact sets as $k \rightarrow \infty$. Since the second term in the above inequality tends to zero uniformly on compact sets we have $f_{1 k}(t)$ tends to $\omega\left(A \alpha_{t}(B)\right)$ uniformly for $t$ in a compact set. A similar calculation shows that $f_{2 k}(t)$ tends to $\omega\left(\alpha_{t}(B) A\right)$ uniformly for $t$ in a compact set. It follows from the integral representation of $F_{k}(z), 0<\operatorname{Im}(z)<\beta$, and the facts that $f_{1 k}$ and $f_{2 k}$ are uniformly bounded [in fact, $\left|f_{1 k}(t)\right| \leqq\|A\|\|B\|$ and $\left|f_{2 k}(t)\right| \leqq\|A\|\|B\|$ for all real $t$ and $\left.k=1,2, \ldots\right]$ and $\left\|K_{1}(\cdot, z)\right\|_{1}$ $+\left\|K_{2}(\cdot, z)\right\|_{1}=1$, that $F_{k}(z)$ converges to an analytic function $F(z)$ which is holomorphic for $0<\operatorname{Im}(z)<\beta$ and bounded and continuous for $0 \leqq \operatorname{Im}(z) \leqq \beta$ and the convergence is uniform on compact subsets of the strip $0 \leqq \operatorname{Im}(z) \leqq \beta$.

Since $F(t)=\omega\left(A \alpha_{t}(B)\right)$ and $F(t+i \beta)=\omega\left(\alpha_{t}(B) A\right)$ it follows that $\omega$ satisfies the KMS condition. This completes the proof of the theorem.

Remark. Since for quantum lattice systems the dynamics is given by an approximately inner one parameter group of *-automorphisms (see ([14], p. 193), [13] and [1]) and since the $C^{*}$-algebra describing quantum lattice systems have trace states it follows that these systems have KMS states for all inverse temperatures $\beta>0$. Actually, we have KMS states exist for all inverse temperatures both positive and negative since the automorphism group $\left\{\alpha_{t}^{\prime}=\alpha_{-t}\right\}$ is approximately inner if and only if $\left\{\alpha_{t}\right\}$ is approximately inner. It is the usual convention to define KMS states only for positive temperatures. 


\section{Unbounded Dérivations of UHF Algebras}

A uniformly hyperfinite (UHF) algebra is a $C^{*}$-algebra $\mathfrak{A}$ which contains an increasing sequence $M_{1} \subset M_{2} \subset \cdots$ of $\left(n_{i} \times n_{i}\right)$-matrix algebras whose union $\mathfrak{A}_{0}=\bigcup_{i=1}^{\infty} M_{i}$ is a norm dense *-subalgebra of $\mathfrak{A}$. UHF algebras were introduced and studied by Glimm [5].

Suppose $\left\{\alpha_{t}\right\}$ is a strongly continuous one parameter group of *-automorphisms of a UHF algebra $\mathfrak{A}$ and $\delta$ is the derivation which generates $\left\{\alpha_{t}\right\}$. An element $A \in \mathfrak{U}$ is said to be an analytic element for $\delta$ or $\left\{\alpha_{t}\right\}$ if the function $t \rightarrow \alpha_{t}(A)$ can be extended to an analytic function in the strip $|\operatorname{Im}(z)|<r$ with $z=t+i y$ and $r>0$. It follows from Nelson's paper [11] that an element $A \in \mathfrak{A}$ is an analytic element if and only if $A \in \mathfrak{D}(\delta), \delta(A) \in \mathfrak{D}(\delta), \delta(\delta(A))=\delta^{2}(A) \in \mathfrak{D}(\delta), \ldots$ and

$$
\sum_{n=0}^{\infty} \frac{s^{n}}{n !}\left\|\delta^{n}(A)\right\|<\infty
$$

for all $0 \leqq s \leqq r$ with $r>0$.

Recently, it was shown in [17] that if $\left\{\alpha_{t}\right\}$ is a strongly continuous one parameter group of $*$-automorphisms of a UHF algebra $\mathfrak{A}$ and $\delta$ is the derivation which generates $\left\{\alpha_{t}\right\}$, then there exists an increasing sequence $M_{1} \subset M_{2} \subset \cdots \subset \mathfrak{U}$ of $\left(n_{1} \times n_{1}\right)$-matrix algebras whose union $\mathfrak{U}_{0}=\bigcup_{i=1}^{\infty} M_{i}$ is a norm dense *-subalgebra of $\mathfrak{U}$ and furthermore, each element $A \in \mathfrak{U}_{0}$ (i.e., $A \in M_{n}$ for some integer $n$ ) is an analytic element for $\delta$. Furthermore, for each matrix algebra $M_{n}$ there is an hermitian element $H_{n} \in \mathfrak{U}$ so that $\delta(A)=i\left[H_{n}, A\right]$ for all $A \in M_{n}$. It follows that if $A \in \mathfrak{U}_{0}$ we have $\delta(A)=\lim _{n \rightarrow \infty} i\left[H_{n}, A\right]$. We will show that if $\mathfrak{A}_{0}$ is a core for $\delta$ then $\left\{\alpha_{t}\right\}$ is approximately inner. First we consider the question of when a *-derivation of $\mathfrak{U}_{0}$ into $\mathfrak{A}$ uniquely defines a one parameter group of *-automorphisms.

Theorem 4.1. Suppose $\mathfrak{A}$ is a UHF algebra and $M_{1} \subset M_{2} \subset \cdots \subset \mathfrak{A}$ is an increasing sequence of $\left(n_{i} \times n_{i}\right)$-matrix algebras whose union $\mathfrak{U}_{0}=\bigcup_{i=1} M_{i}$ is a norm dense *-subalgebra of $\mathfrak{U}$. Suppose $\delta$ is a $*$-derivation of $\mathfrak{U}_{0}$ into $\mathfrak{A}$, i.e., $\delta$ is a linear mapping of $\mathfrak{U}_{0}$ into $\mathfrak{A}$ with the properties

(i) $\delta(A B)=\delta(A) B+A \delta(B)$ for $A, B \in \mathfrak{U}_{0}$.

(ii) $\delta\left(A^{*}\right)=\delta(A)^{*}$ for $A \in \mathfrak{U}_{0}$.

Then, $\delta$ is closable, i.e., there is a unique closed derivation $\bar{\delta}$ with domain $\mathfrak{D}(\bar{\delta}) \supset \mathfrak{U}_{0}$ so that $\bar{\delta}(A)=\delta(A)$ for all $A \in \mathfrak{U}_{0}$ and for all $A \in \mathfrak{D}(\bar{\delta})$ there is a sequence $A_{n} \in \mathfrak{U}_{0}$ so that $\left\|A_{n}-A\right\| \rightarrow 0$ and $\left\|\bar{\delta}(A)-\delta\left(A_{n}\right)\right\| \rightarrow 0$ as $n \rightarrow \infty$. Furthermore, $\bar{\delta}$ is the generator of a strongly continuous one 
parameter group of *-automorphisms of $\mathfrak{U}$ if and only if the only norm continuous linear functionals $\varrho_{ \pm} \in \mathfrak{U}^{*}$ satisfying the equations

$$
\begin{aligned}
& \varrho_{+}(A+\delta(A))=0 \\
& \varrho_{-}(A-\delta(A))=0
\end{aligned}
$$

for all $A \in \mathfrak{U}_{0}$ are the zero functionals $\varrho_{+}=\varrho_{-}=0$.

Proof. Suppose $\delta$ is a $*$-derivation of $\mathfrak{A}_{0}$ into $\mathfrak{U}$. First we show $\delta$ is closable. To show $\delta$ is closable it is sufficient to show that if $A_{n} \in \mathfrak{U}_{0}$ and $\left\|\delta\left(A_{n}\right)-B\right\| \rightarrow 0$ and $\left\|A_{n}\right\| \rightarrow 0$ as $n \rightarrow \infty$ then $B=0$.

Let $\tau$ be the unique trace state of $\mathfrak{A}$ i.e., $\tau(A B)=\tau(B A)$ for all $A, B \in \mathfrak{U}$ and $\tau$ is a state of $\mathfrak{A}$. We will show that $\tau(\delta(A))=0$ for all $A \in \mathfrak{A}_{0}$. Let $\left\{e_{i j}^{(n)} ; i, j=1, \ldots, m(n)\right\}$ be a family of matrix units which span $M_{n} \subset \mathfrak{A}_{0}$. Let $H_{n}$ be the hermitian element of $\mathfrak{A}$ given by

$$
H_{n}=(-i / m(n)) \sum_{i, j=1}^{m(n)} \delta\left(e_{i j}^{(n)}\right) e_{j i}^{(n)} .
$$

A straight forward computation shows $\delta(A)=i\left[H_{n}, A\right]$ for all $A \in M_{n}$. Hence, we have for $A \in M_{n} \tau(\delta(A))=i \tau\left(\left[H_{n}, A\right]\right)=0$. Since $\mathfrak{X}_{0}=\bigcup_{n=1}^{\infty} M_{n}$ we have $\tau(\delta(A))=0$ for all $A \in \mathfrak{A}_{0}$.

We define an inner product $(A, B)=\tau\left(A^{*} B\right)$ on $\mathfrak{A}$. Let $\mathfrak{H}$ be the Hilbert space obtained by completing $\mathfrak{U}$ with respect to this inner product. We consider $\mathfrak{U}_{0} \subset \mathfrak{U} \subset \mathfrak{H}$ as dense subsets of $\mathfrak{H}$. Consider the linear operator $\Gamma$ from $\mathfrak{U}_{0}$ into $\mathfrak{H}$ given by $\Gamma A=i \delta(A)$ for $A \in \mathfrak{A}_{0}$. We have $\Gamma$ is hermitian since for $A, B \in \mathfrak{U}_{0}$ we have

$$
\begin{aligned}
& (A, \Gamma B)=\tau\left(A^{*} i \delta(B)\right)=i \tau\left(A^{*} \delta(B)\right) \\
& (\Gamma A, B)=\tau\left((i \delta(A))^{*} B\right)=-i \tau\left(\delta\left(A^{*}\right) B\right)
\end{aligned}
$$

and

$$
(A, \Gamma B)-(\Gamma A, B)=i \tau\left(A^{*} \delta(B)+\delta\left(A^{*}\right) B\right)=i \tau\left(\delta\left(A^{*} B\right)\right)=0 .
$$

Since $\Gamma$ is hermitian the hermitian adjoint $\Gamma^{*}$ is densely defined and, therefore, $\Gamma$ is closable (see [12], p. 305 and 306). Hence, if $A_{n} \rightarrow 0$ and $\Gamma A_{n} \rightarrow F \in \mathfrak{H}$ in the Hilbert space topology we have $F=0$. Now suppose $A_{n} \in \mathfrak{U}_{0},\left\|\delta\left(A_{n}\right)-B\right\| \rightarrow 0$ and $\left\|A_{n}\right\| \rightarrow 0$ as $n \rightarrow \infty$ with $B \in \mathfrak{U}$, then $A_{n} \rightarrow 0$ and $\Gamma A_{n} \rightarrow i B$ in the norm topology of $\mathfrak{H}$. Hence, $B=0$. Hence, $\delta$ is closable.

Let $\bar{\delta}$ be the closure of $\delta$ and let $\mathfrak{D}(\bar{\delta})$ be the domain of $\bar{\delta}$. We will show $\bar{\delta}$ is a $*$-derivation of $\mathfrak{D}(\bar{\delta})$ into $\mathfrak{A}$. Suppose $A \in \mathfrak{D}(\bar{\delta})$. Then there is a sequence $\left\{A_{n} \in \mathfrak{U}_{0}\right\}$ so that $\left\|A_{n}-A\right\| \rightarrow 0$ and $\left\|\delta\left(A_{n}\right)-\bar{\delta}(A)\right\| \rightarrow 0$ as $n \rightarrow \infty$. Hence, $\left\|A_{n}^{*}-A^{*}\right\| \rightarrow 0$ and $\| \delta\left(A_{n}^{*}\right)$ $-\delta(A)^{*} \| \rightarrow 0$ as $n \rightarrow \infty$. Since $\bar{\delta}$ is closed we have $A^{*} \in \mathfrak{D}(\bar{\delta})$ 
and $\bar{\delta}\left(A^{*}\right)=\bar{\delta}(A)^{*}$. Next, suppose $A, B \in \mathfrak{D}(\bar{\delta})$. Then there are sequences $\left\{A_{n}, B_{n} \in \mathfrak{A}_{0}\right\} \quad$ so that $\quad\left\|A_{n}-A\right\| \rightarrow 0, \quad\left\|B_{n}-B\right\| \rightarrow 0$, $\left\|\delta\left(A_{n}\right)-\bar{\delta}(A)\right\| \rightarrow 0$ and $\left\|\delta\left(B_{n}\right)-\bar{\delta}(B)\right\| \rightarrow 0$ as $n \rightarrow \infty$. Hence, we have $\left\|A_{n} B_{n}-A B\right\| \rightarrow 0$ and $\left\|\delta\left(A_{n} B_{n}\right)-(\bar{\delta}(A) B+A \bar{\delta}(B))\right\| \rightarrow 0$ as $n \rightarrow \infty$. Hence, $A B \in \mathfrak{D}(\bar{\delta})$ and $\bar{\delta}(A B)=\bar{\delta}(A) B+A \bar{\delta}(B)$. Hence, $\bar{\delta}$ is a closed $*$-derivation of $\mathfrak{D}(\bar{\delta})$ into $\mathfrak{U}$.

Next, we will show $\bar{\delta}$ is the generator of a strongly continuous one parameter group of $*$-automorphisms of $\mathfrak{A}$ if and only if the only norm continuous linear functionals $\varrho_{ \pm}$satisfying the equations $\varrho_{+}(A+\delta(A))=0$ and $\varrho_{-}(A-\delta(A))=0$ for all $A \in \mathfrak{A}_{0}$ are the zero functionals.

First, suppose $\bar{\delta}$ is the generator of a strongly continuous one parameter group of $*$-automorphisms of $\mathfrak{A}$. Suppose $\varrho_{+}$is a norm continuous linear functional on $\mathfrak{A}$ and $\varrho_{+}(A+\delta(A))=0$ for all $A \in \mathfrak{A}_{0}$. Since $\mathfrak{A}_{0}$ is a core for $\bar{\delta}$ we have for all $A \in \mathfrak{D}(\bar{\delta})$ there is a sequence $\left\{A_{n} \in \mathfrak{A}_{0}\right\}$ so that $\left\|A_{n}-A\right\| \rightarrow 0$ and $\left\|\delta\left(A_{n}\right)-\bar{\delta}(A)\right\| \rightarrow 0$ as $n \rightarrow \infty$. Hence, we have $\varrho_{+}(A+\bar{\delta}(A))=\lim _{n \rightarrow \infty} \varrho_{+}\left(A_{n}+\delta\left(A_{n}\right)\right)=0$. Hence, $\varrho_{+}(A+\bar{\delta}(A))=0$ for all $A \in \mathfrak{D}(\bar{\delta})$. For $A \in \mathfrak{D}(\bar{\delta})$ we have

$$
\frac{d}{d t} \varrho_{+}\left(\alpha_{t}(A)\right)=\varrho_{+}\left(\delta\left(\alpha_{t}(A)\right)\right)=-\varrho_{+}\left(\alpha_{t}(A)\right) .
$$

Hence, $\varrho_{+}\left(\alpha_{t}(A)\right)=e^{-t} \varrho_{+}(A)$ for all $A \in \mathfrak{D}(\bar{\delta})$, and all real $t$. Since $\left|\varrho_{+}\left(\alpha_{t}(A)\right)\right| \leqq\left\|\varrho_{+}\right\|\left\|\alpha_{t}(A)\right\|=\left\|\varrho_{t}\right\|\|A\|$ for all real $t$ and $e^{-t}$ grows without bound as $t \rightarrow-\infty$ we have $\varrho_{+}(A)=0$ for all $A \in \mathfrak{D}(\bar{\delta})$. Since $\varrho_{+}$is continuous and $\mathfrak{D}(\bar{\delta})$ is dense in $\mathfrak{A}$ it follows $\varrho_{+}=0$. A similar argument shows $\varrho_{-}=0$. Hence, if $\bar{\delta}$ is the generator of a strongly continuous one parameter group of $*$-automorphisms the functionals $\varrho_{ \pm}$are necessarily zero.

Now, suppose $\delta$ is a $*$-derivation of $\mathfrak{A}_{0}$ into $\mathfrak{A}$ with the property that the only norm continuous linear functionals $\varrho_{ \pm}$on $\mathfrak{U}$ satisfying the equations $\varrho_{+}(A+\delta(A))=0$ and $\varrho_{-}(A-\delta(A))=0$ for all $A \in \mathfrak{A}_{0}$ are the zero functionals. We will show that $\bar{\delta}$ the closure of $\delta$ is the generator of a strongly continuous one parameter group of $*$-automorphisms of $\mathfrak{A}$.

It follows from semi-group theory (see [3] or [8]) that $\bar{\delta}$ is the generator of strongly continuous contraction semi-group if and only if the mapping $A \rightarrow \lambda A-\bar{\delta}(A)$ from $\mathfrak{D}(\bar{\delta})$ into $\mathfrak{A}$ is one to one and has range all of $\mathfrak{A}$ and the norm of the inverse mapping (consider as a linear mapping of the Banach space $\mathfrak{U}$ into itself) satisfies the relation $\left\|(\lambda-\bar{\delta})^{-1}\right\| \leqq \lambda^{-1}$ for all $\lambda>0$. If $\bar{\delta}$ is the generator of a one parameter group of $*$-automorphisms (i.e., the automorphisms $\alpha_{t}$ exist for both positive and negative $t$ ), then both $\bar{\delta}$ and $-\bar{\delta}$ are generators of contraction semigroups. Hence, $\bar{\delta}$ generates a one parameter group of $*$-automorphisms if $A \rightarrow \lambda A \pm \bar{\delta}(A)$ are one to one mappings of $\mathfrak{D}(\bar{\delta})$ onto $\mathfrak{A}$ and $\left\|(\lambda \pm \bar{\delta})^{-1}\right\|$ $\leqq \lambda^{-1}$ for all $\lambda>0$. 
A straight forward computation shows that these conditions on $\bar{\delta}$ are equivalent to the conditions $\|A+\lambda \bar{\delta}(A)\| \geqq\|A\|$ for all $A \in \mathfrak{D}(\bar{\delta})$ and the range of the maps $A \rightarrow A+\lambda \delta(A)$ of $\mathfrak{D}(\bar{\delta})$ into $\mathfrak{U}$ is all of $\mathfrak{A}$ for real $\lambda \neq 0$.

We will begin by showing $\|A+\lambda \delta(A)\| \geqq\|A\|$ for all $A \in \mathfrak{U}_{0}$ and all real $\lambda$. Suppose $A \in \mathfrak{U}_{0}$ and $\lambda$ is real. There is an integer $n$ so that $A \in M_{n}$ and there is an hermitian element $H_{n} \in \mathfrak{U}$ so that $\delta(B)=i\left[H_{n}, B\right]$ for all $B \in M_{n}$. Since $A \in M_{n}$ there is a state $\omega$ of $M_{n}$ so that $\omega\left(A^{*} A\right)=\left\|A^{*} A\right\|$ $=\|A\|^{2}$. It follows from the Hahn-Banach theorem that $\omega$ has an extension which we also denote by $\omega$ to a state on all of $\mathfrak{A}$. Now, we have

$$
\begin{aligned}
\omega((A+\lambda \delta(A)) * & (A+\lambda \delta(A))) \\
= & \omega\left(A^{*} A\right)+\lambda \omega\left(\delta\left(A^{*} A\right)\right)+|\lambda|^{2} \omega\left(\delta(A)^{*} \delta(A)\right) \\
& =\omega\left(A^{*} A\right)+i \lambda \omega\left(\left[H_{n}, A^{*} A\right]\right)+|\lambda|^{2} \omega\left(\delta(A)^{*} \delta(A)\right)
\end{aligned}
$$

Since $D=\|A\|^{2} I-A^{*} A \geqq 0$ and $\omega(D)=0$ it follows from the generalized Schwarz inequality that

$$
\begin{aligned}
& |\omega(B D)|^{2}=\left|\omega\left(B D^{1 / 2} D^{1 / 2}\right)\right|^{2} \leqq \omega\left(B D B^{*}\right) \omega(D)=0 \\
& |\omega(D B)|^{2}=\left|\omega\left(D^{1 / 2} D^{1 / 2} B\right)\right|^{2} \leqq \omega(D) \omega\left(B^{*} D B\right)=0
\end{aligned}
$$

for all $B \in \mathfrak{U}$. Hence, we have

$$
\omega\left(\left[H_{n}, A^{*} A\right]\right)=-\omega\left(\left[H_{n}, D\right]\right)=\omega\left(D H_{n}\right)-\omega\left(H_{n} D\right)=0-0=0 .
$$

Since $\omega\left(\left[H_{n}, A^{*} A\right]\right)=0$ we have

$$
\begin{aligned}
\omega\left((A+\lambda \delta(A))^{*}(A+\lambda \delta(A))\right) & =\omega\left(A^{*} A\right)+|\lambda|^{2} \omega\left(\delta(A)^{*} \delta(A)\right) \\
& =\|A\|^{2}+|\lambda|^{2} \omega\left(\delta(A)^{*} \delta(A)\right) \\
& \geqq\|A\|^{2} .
\end{aligned}
$$

Since $\omega\left(B^{*} B\right) \leqq\|B\|^{2}$ for all $B \in \mathfrak{U}$ we have $\|A+\lambda \delta(A)\| \geqq\|A\|$ for all $A \in \mathfrak{U}_{0}$ and $\lambda$ real. Since $\mathfrak{U}_{0}$ is a core for $\bar{\delta}$ it follows that $\|A+\lambda \bar{\delta}(A)\| \geqq\|A\|$ for all $A \in \mathfrak{D}(\bar{\delta})$ and $\lambda$ real. It follows that if $(I+\lambda \bar{\delta})^{-1}$ exists then $\left\|(I+\lambda \bar{\delta})^{-1}\right\| \leqq 1$ for all real $\lambda$.

Since $\|A+\lambda \bar{\delta}(A)\| \geqq\|A\|$ for all $A \in \mathfrak{D}(\bar{\delta})$ and $\lambda$ real and since $\bar{\delta}$ is closed a straight forward computation shows the range of the map $A \rightarrow A+\lambda \bar{\delta}(A), A \in \mathfrak{D}(\delta)$ is norm closed for $\lambda$ real and $\lambda \neq 0$. If the range of this mapping is not all of $\mathfrak{A}$ then it follows from the Hahn-Banach theorem there is a non zero norm continuous linear functional $\varrho_{\lambda}$ on $\mathfrak{U}$ so that $\varrho_{\lambda}(A+\lambda \bar{\delta}(A))=0$ for all $A \in \mathfrak{D}(\bar{\delta})$. By assumption we have the only norm continuous solutions to the equations $\varrho_{+}(A+\delta(A))=0$ and $\varrho_{-}(A-\delta(A))=0$ for all $A \in \mathfrak{U}_{0}$ are the functionals $\varrho_{+}=\varrho_{-}=0$. Hence, it follows that the mappings $A \rightarrow A \pm \bar{\delta}(A), A \in \mathfrak{D}(\bar{\delta})$ have range all of $\mathfrak{U}$. Since these mappings are norm increasing we have $(I \pm \bar{\delta})^{-1}$ 
exist and $\left\|(I \pm \bar{\delta})^{-1}\right\| \leqq 1$. From the resolvent equation $A^{-1}-B^{-1}$ $=A^{-1}(B-A) B^{-1}$ and solving for $B=\left(I+A^{-1}(B-A)\right)^{-1} A^{-1}$ [valid when $\left.\left\|A^{-1}(B-A)\right\|<1\right]$ we have

$$
\begin{aligned}
& (\lambda+\bar{\delta})^{-1}=\left(I+(\lambda-1)(I+\bar{\delta})^{-1}\right)^{-1}(I+\bar{\delta})^{-1} \\
& (\lambda-\bar{\delta})^{-1}=\left(I+(\lambda-1)(I-\bar{\delta})^{-1}\right)^{-1}(I-\bar{\delta})^{-1}
\end{aligned}
$$

for all $\lambda$ so that $|\lambda-1|<1$ since then $\left\|(\lambda-1)(I \pm \bar{\delta})^{-1}\right\| \leqq|\lambda-1|<1$ and then $I+(\lambda-1)(I \pm \bar{\delta})^{-1}$ is invertable. Hence, we have $(\lambda \pm \bar{\delta})^{-1}$ exists for $0<\lambda<2$. Since $\|A+\lambda \bar{\delta}(A)\| \geqq\|A\|$ for all $A \in \mathfrak{D}(\bar{\delta})$ and $\lambda$ real it follows $\|\lambda A \pm \bar{\delta}(A)\| \geqq \lambda\|A\|$ for all $\lambda>0$ and $A \in \mathfrak{D}(\bar{\delta})$. Hence, $\left\|(\lambda \pm \bar{\delta})^{-1}\right\|$ $\leqq \lambda^{-1}$ for $0<\lambda<2$. Using the resolvent equation again we have

$$
\begin{aligned}
& \left(\lambda^{\prime}+\bar{\delta}\right)^{-1}=\left(I+\left(\lambda^{\prime}-\lambda\right)(\lambda+\bar{\delta})^{-1}\right)^{-1}(\lambda+\bar{\delta})^{-1} \\
& \left(\lambda^{\prime}-\bar{\delta}\right)^{-1}=\left(I+\left(\lambda^{\prime}-\lambda\right)(\lambda-\bar{\delta})^{-1}\right)^{-1}(\lambda-\bar{\delta})^{-1}
\end{aligned}
$$

provided $\left\|\left(\lambda^{\prime}-\lambda\right)(\lambda \pm \bar{\delta})^{-1}\right\| \leqq\left|\left(\lambda^{\prime}-\lambda\right) / \lambda\right|<1$. Setting $\lambda=2-\varepsilon$ the above equations show that $\left(\lambda^{\prime} \pm \overline{\bar{\delta}}\right)^{-1}$ exist for $\left|\left(\lambda^{\prime}-(2-\varepsilon)\right) /(2-\varepsilon)\right|<1$ or $0<\lambda^{\prime}<4-2 \varepsilon$. Hence, $(\lambda \pm \overline{\bar{\delta}})^{-1}$ exists for $0<\lambda<4$ and $\left\|(\lambda+\bar{\delta})^{-1}\right\| \leqq \lambda^{-1}$. Continuing in this manner we find $(\lambda \pm \bar{\delta})^{-1}$ exists for all $\lambda>0$ and $\left\|(\lambda \pm \bar{\delta})^{-1}\right\| \leqq \lambda^{-1}$. Hence, from the general theory of semi-groups $\bar{\delta}$ and $-\bar{\delta}$ are generators of contraction semi-groups or equivalently $\bar{\delta}$ is the generator of a strongly continuous group of contractions $\left\{\alpha_{t}\right\}$.

We complete the proof of the theorem by showing $\left\{\alpha_{t}\right\}$ is a group of *-automorphisms. Let $\beta_{t}(A)=\alpha_{t}\left(A^{*}\right)^{*}$ for all $A \in \mathfrak{A}$ and all real $t$. We have $\left\{\beta_{t}\right\}$ is a strongly continuous group of contractions of $\mathfrak{A}$ into $\mathfrak{A}$. The generator of $\left\{\beta_{t}\right\}$ is the operator $\delta_{1}(A)=\delta\left(A^{*}\right)^{*}=\delta(A)$ for all $A \in \mathfrak{D}(\bar{\delta})$. Hence, $\alpha_{t}=\beta_{t}$ for all real $t$ and, hence, $\alpha_{t}\left(A^{*}\right)=\alpha_{t}(A)^{*}$ for all $A \in \mathfrak{U}$.

Next, suppose $A, B \in \mathfrak{D}(\bar{\delta})$ and let $C(t)=\alpha_{-t}\left(\alpha_{t}(A) \alpha_{t}(B)\right)$. Since $\alpha_{t}$ maps $\mathfrak{D}(\bar{\delta})$ into $\mathfrak{D}(\bar{\delta})$ and since $\bar{\delta}$ is a $*$-derivation $\alpha_{t}(A) \alpha_{t}(B) \in \mathfrak{D}(\bar{\delta})$. A straight forward calculation then shows $d C(t) / d t=0$ for all real $t$ where the derivative exists in the sense of norm convergence. Hence $\alpha_{-t}\left(\alpha_{t}(A) \alpha_{t}(B)\right)=A B$ and $\alpha_{t}(A B)=\alpha_{t}(A) \alpha_{t}(B)$ for all $A, B \in \mathfrak{D}(\bar{\delta})$. Since the $\alpha_{t}$ are contractions and $\mathfrak{D}(\bar{\delta})$ is dense in $\mathfrak{U}$ we have $\alpha_{t}(A B)=\alpha_{t}(A) \alpha_{t}(B)$ for all $A, B \in \mathfrak{A}$. Hence, $\left\{\alpha_{t}\right\}$ is a strongly continuous one parameter group of $*$-automorphisms of $\mathfrak{A}$. This completes the proof of the theorem.

Remark. Theorem 4.1 shows that $\bar{\delta}$ is the generator of a strongly continuous one parameter group of $*$-automorphisms of $\mathfrak{A}$ if and only if the sets $S_{ \pm}=\left\{A \pm \delta(A) ; A \in \mathfrak{U}_{0}\right\}$ are norm dense in $\mathfrak{A}$. The result remains true if the sets $S_{ \pm}$are replaced by the sets $S_{ \pm}^{\prime}=\{\lambda A \pm \delta(A)$; $\left.A \in \mathfrak{U}_{0}\right\}$ with the real part of $\lambda$ not equal to zero.

If $T$ is a densely defined hermitian operator on a Hilbert space $\mathfrak{H}$ then $T$ has a self-adjoint extension $T_{1}$ if and only if the dimension of $\mathfrak{D}_{+}$ equals the dimension of $\mathfrak{D}_{-}$where $\mathfrak{D}_{+}=\{\operatorname{Range}(T+i I)\}^{\perp}$ and 
$\mathfrak{D}_{-}=\{\operatorname{Range}(T-i I)\}^{\perp}$. It would be interesting to know under what conditions a $*$-derivation $\delta$ of $\mathfrak{A}_{0}$ into $\mathfrak{U}$ has an extension $\delta_{1}$ which is the generator of a strongly continuous one parameter group of *-automorphisms.

Theorem 4.2. Suppose $\mathfrak{A}$ is a UHF algebra and $M_{1} \subset M_{2} \subset \cdots \subset \mathfrak{A}$ is an increasing sequence of $\left(n_{i} \times n_{i}\right)$-matrix algebras whose union $\mathfrak{U}_{0}=\bigcup_{i=1}^{\infty} M_{i}$ is a norm dense *-subalgebra of $\mathfrak{A}$. Suppose $\delta$ is a $*$-derivation of $\mathfrak{\mathfrak { V }}_{0}$ into $\mathfrak{A}$ whose closure $\bar{\delta}$ is the generator of a strongly continuous one parameter group of *-automorphisms $\left\{\alpha_{t}\right\}$. Then, the automorphism group $\left\{\alpha_{t}\right\}$ is approximately inner.

Proof. Suppose the hypothesis of the theorem is true. Let $\left\{e_{i j}^{(n)}\right.$; $i, j=1, \ldots, m(n)\}$ be a family of matrix units which span $M_{n}$ and let

$$
H_{n}=(-i / m(n)) \sum_{1,1=1}^{m(n)} \delta\left(e_{i j}^{(n)}\right) e_{j i}^{(n)} .
$$

Let $\delta_{n}(A)=i\left[H_{n}, A\right]$ for all $A \in \mathfrak{A}$. We have $\delta_{n}$ is an inner $*$-derivation of $\mathfrak{U}$ into $\mathfrak{A}$ and $\delta_{n}(A)=\delta(A)$ for all $A \in M_{n}$. We will show that $\left(I-\delta_{n}\right)^{-1}$ converges strongly to $(I-\bar{\delta})^{-1}$ as $n \rightarrow \infty$.

Since $\bar{\delta}$ is the generator of $\left\{\alpha_{t}\right\}$ we have $(I-\bar{\delta})^{-1}$ exists. In fact, we have

$$
(I-\bar{\delta})^{-1}(A)=\int_{0}^{\infty} e^{-t} \alpha_{t}(A) d t
$$

for all $A \in \mathfrak{A}$. Hence, the range of the map $A \rightarrow A-\bar{\delta}(A), A \in \mathfrak{D}(\bar{\delta})$, is all of $\mathfrak{U}$. Since $\mathfrak{U}_{0}$ is a core for $\bar{\delta}$ (i.e., $\bar{\delta}$ is the closure of its restriction to $\mathfrak{U}_{0}$ ) the set $S_{-}=\left\{A-\bar{\delta}(A) ; A \in \mathfrak{U}_{0}\right\}$ is norm dense in $\mathfrak{A}$. Suppose $A \in S_{-}$. We have $A=B-\bar{\delta}(B)$ with $B \in \mathfrak{U}_{0}$ and

$$
\begin{aligned}
\|\left(I-\delta_{n}\right)^{-1}(A) & -(I-\bar{\delta})^{-1}(A) \| \\
& =\left\|\left\{\left(I-\delta_{n}\right)^{-1}\left(\bar{\delta}-\delta_{n}\right)(I-\bar{\delta})^{-1}\right\}(A)\right\| \\
& =\left\|\left\{\left(I-\delta_{n}\right)^{-1}\left(\bar{\delta}-\delta_{n}\right)(I-\bar{\delta})^{-1}(I-\bar{\delta})\right\}(B)\right\| \\
& =\left\|\left(I-\delta_{n}\right)^{-1}\left(\delta(B)-\delta_{n}(B)\right)\right\| \\
& \leqq\left\|\delta(B)-\delta_{n}(B)\right\| \rightarrow 0
\end{aligned}
$$

as $n \rightarrow \infty$ since $\left\|\left(I-\delta_{n}\right)^{-1}\right\| \leqq 1$ and $B \in \mathfrak{U}_{0}$. Hence, for $A \in S_{-}\left(I-\delta_{n}\right)^{-1}(A)$ converges in norm to $(I-\bar{\delta})^{-1}(A)$ as $n \rightarrow \infty$. Since $\left\|(I-\bar{\delta})^{-1}\right\| \leqq 1$ and $\left\|\left(I-\delta_{n}\right)^{-1}\right\| \leqq 1$ for all $n=1,2, \ldots$ and $S_{-}$is norm dense in $\mathfrak{U}$ we have $\left(I-\delta_{n}\right)^{-1}(A)$ converges to $(I-\bar{\delta})^{-1}(A)$ for all $A \in \mathfrak{A}$.

Since $\left(I-\delta_{n}\right)^{-1}$ converges strongly to $(I-\bar{\delta})^{-1}$ we have by the Trotter convergence theorem (see [21] or ([9], p. 502))

$$
\begin{aligned}
\alpha_{t}(A) & =\{\exp (t \bar{\delta})\}(A)=\lim _{n \rightarrow \infty}\left\{\exp \left(t \delta_{n}\right)\right\}(A) \\
& =\lim _{n \rightarrow \infty} e^{i t H_{n}} A e^{-t t H_{n}},
\end{aligned}
$$


for all $A \in \mathfrak{A}$. Hence, $\left\{\alpha_{t}\right\}$ is approximately inner. This completes the proof of the theorem.

Conjecture. We conjecture that every strongly continuous one parameter group of $*$-automorphisms of a UHF algebra is approximately inner. The truth of this conjecture would show that every strongly continuous one parameter group of *-automorphisms of a UHF algebra has a ground state and a KMS state for all inverse temperatures $\beta$.

\section{References}

1. Araki,H., Ion,P.D.F.: On the equivalence of KMS and Gibbs conditions for states of quantum lattice systems. Preprint

2. Borchers, H.: Commun. math. Phys. 2, 49-54 (1966)

3. Dunford, N., Schwartz,J.T.: Linear Operators, Part I. New York: Interscience Pub. 1963

4. Gelfand, M., Vilenkin, Y.: Generalized functions, Vol. I and IV. New York: Academic Press 1964

5. Glimm, J.: Trans. Amer. Math. Soc. 95, 318-340 (1960)

6. Haag, R., Hugenholtz, N. M., Winnink, M.: Commun. math. Phys. 5, 215-236 (1967)

7. Hille, E.: Analytic function theory, Vol. II. New York: Ginn and Co., 1962

8. Hille, E., Phillips, R.S.: Functional analysis and semigroups. American Mathematical Society Colloquium Publications (revised ed.), Vol. 31. Providence, Rhode Island (1957)

9. Kato, T.: Perturbation Theory for linear operators. Berlin-Heidelberg-New York: Springer 1966

10. Naimark, M.A.: Normed rings. In: Groningen: P. Noordhoff 1964

11. Nelson, E.: Ann. Math. 70, 572-613 (1959)

12. Riesz,F., Nagy, S.: Functional analysis. New York: Ungar Pub. Co., 1955

13. Robinson, D. W.: I. Commun. math. Phys., 6, 151-160 (1967). II. Commun. math. Phys. 7, 337-348 (1968)

14. Ruelle, D.: Statistical mechanics. New York: W. A. Benjamin, Inc., 1969

15. Ruelle, O.: Commun. math. Phys., 11, 339-345 (1969)

16. Sakai, S.: $C^{*}$-algebras and $W^{*}$-algebras. Berlin-Heidelberg-New York: Springer 1971

17. Sakai, S.: On one-parameter subgroups of ${ }^{*}$-automorphisms on operator algebras and the corresponding unbounded derivations. Preprint

18. Schwartz, L.: Theorie des distributions. Paris: Hermann. Part I 1957; Part II 1959

19. Shale, D.: Math. Phys. 3, 915-921 (1962)

20. Streater, R.F., Wightman, A.S.: PCT, spin, and statistics, and all that. New York: W. A. Benjamin Inc., 1964

21. Trotter,H.F.: Pacific J. Math. 8, 887-919 (1958)

Communicated by A. S. Wightman

\section{R. T. Powers}

Mathematics Department

University of Pennsylvania

Philadelphia, Pa. 19104, USA 